recibido: 18.09 .12 / aceptado: 11.04 .13

\title{
El empoderamiento digital de niños y jóvenes a través de la producción audiovisual
}

José Ignacio Aguaded Gómez

Universidad de Huelva

Jacqueline Sánchez Carrero

Universidad de Huelva

\section{Palabras clave}

Empoderamiento, infancia, juventud, producción audiovisual, lectura crítica, educomunicación.

\section{Resumen}

Los niños, adolescentes y jóvenes del presente siglo están transitando su andadura por la producción audiovisual de modo cada vez más práctico. Reclaman un espacio propio haciendo oír sus voces en el mundo digital. La capacidad que están desarrollan no solo a través de la producción audiovisual sino también en la recepción crítica de los contenidos de las pantallas genera en ellos importantes descubrimientos acerca de la industria de los medios de comunicación. En este artículo nos adentramos en el concepto de educación mediática describiendo el proceso y el resultado de una investigación realizada con niños de 7 a 11 años de edad acerca del universo que existe detrás de las cámaras de televisión. En la experiencia ellos son creadores, escriben sus guiones, graban con cámara de vídeo, editan digitalmente y se ejercitan, a la vez, en la lectura crítica de dibujos animados, series, películas y anuncios publicitarios. Por último, mostramos el enfoque de la llamada Generación M2, niños y jóvenes que están viviendo un proceso de empoderamiento proporcionado por el uso creativo y participativo de los medios digitales. 


\title{
The digital empowerment of children and youth through audiovisual pro- duction
}

\section{Key words}

Empowerment, children, youth, audiovisual production, critical reading, media education.

\begin{abstract}
Children and young people of this century are making their journey by audiovisual pro-duction which is ever more practical. They claim their own space making their voices heard. The capacity developed through audiovisual production and critical reception generate within them important findings about the media industry. In this article we examine the concept of media literacy and describe the process and outcome of research on learning from television with 7-11 year old children in which they are creators. They write their scripts, record with video cameras, edit digitally and exercise a critical reading of cartoons, TV shows, movies and commercials. And finally, we show the approach of the Generation M2, children and youth who are undergoing a process of empowerment by the creative and participatory use of digital media.
\end{abstract}

\begin{abstract}
Autores
José Ignacio Aguaded Gómez [vicerrector.tecnologias@uhu.es] es vicerrector de Tecnologías y Calidad de la Universidad de Huelva, Investigador Principal del Grupo de Investigación «Ágora» (PAI-HUM-648) del Plan Andaluz de Investigación y director de la Revista Científica Iberoamericana «Comunicar» (www. revistacomunicar.com).

Jacqueline Sánchez Carrero [jsanchezcarrero@gmail.com] es miembro del servicio de Informática y Comunicaciones de la Universidad de Huelva, miembro del Grupo de Investigación «Ágora» (PAI-HUM-648) del Plan Andaluz de Investigación y del Grupo Comunicar. Dirige el Taller de Alfabetización Audiovisual para niños Telekids.
\end{abstract}

\section{Créditos}

Estudio enmarcado en la Convocatoria de Proyectos I+D del Ministerio de Economía y Competitividad con clave: EDU2010-21395-C03-03, titulado «La enseñanza obligatoria ante la competencia en comunicación audiovisual en un entorno digital» y en el Proyecto de Investigación de Excelencia de la Junta de Andalucía SEJ-5823-2010, denominado «La competencia audiovisual de la ciudadanía andaluza. Estrategias de alfabetización mediática en la sociedad del ocio digital». 


\section{Educación mediática: un concepto que nace para el cambio}

Una de las señas de identidad del siglo XXI es la tecnología. No se trata solo de la estela de modernización que deja a su paso en la sociedad en general, sino especialmente el empleo que hacen de ella gran parte de niños y adolescentes alrededor del mundo. De hecho, instituciones internacionales como la UNESCO, la Unión Europea, Unicef, entre otras, están comenzando a dar pasos concretos definiendo el alfabetismo en este sentido y estableciendo líneas de trabajo en la llamada educación mediática.

La media literacy -o educación mediática como es más conocida en Hispanoamérica- ha sido delimitada en diversos ámbitos. En América destaca el del Center for Media Literacy (CML) que la define como aquel tipo de enseñanza que «suministra un marco de referencia para acceder, analizar, evaluar y crear mensajes en una diversidad de formatos (desde impresos hasta videos o Internet) y que conduce a la comprensión del papel que juegan los medios en la sociedad así como a las habilidades esenciales de indagación y autoexpresión necesarias para los ciudadanos de una democracia» ${ }^{1}$. En la esfera europea el primero en definir oficialmente el término -después de varias décadas de intentos por unificar criterios en los distintos países del continente- ha sido el Parlamento Europeo, declarando que la educación mediática consiste en «comprender y valorar críticamente los diversos aspectos de los distintos medios de comunicación, consiguiendo filtrar certeramente la información recibida a través del torrente de datos e imágenes». A la vez, hace referencia al concepto de «producción» indicando la necesidad de capacitar al usuario -niños y adultos- en «la elaboración de productos mediáticos (...) como medida de formación práctica» ${ }^{2}$.

La alfabetización de los medios de comunicación en la era digital ha sido materia de análisis en el Parlamento Europeo, no solo para definirla sino también para señalar otras implicaciones. Apunta que temas como la política de información de los consumidores, los derechos de propiedad intelectual, el diálogo intercultural y la participación activa de los ciudadanos son colindantes con la alfabetización mediática. Es por ello que demarca acciones concretas que han de activarse en los años venideros, tal como contemplan los ítems -del 18 al 27- del citado documento, entre los que destacan los siguientes ${ }^{3}$ :

1 El CML es una organización educativa creada para fomentar la educación, el liderazgo y el desarrollo profesional en los Estados Unidos. Fundada a finales de la década de los ochenta, despunta como uno de las instituciones reconocidas que ofrece un portal en Internet lleno de recursos educativos con el fin de facilitar al usuario la creación, el análisis crítico y la evaluación de los contenidos mediáticos. Puede consultarse en: www.medialit.org

2 Véase en: www.europarl.europa.eu/news/expert/infopress_page/037-44615-350-

12-51-906-20081216IPR44614-15-12-2008-2008-false/default_es.htm.

3 Léase la Resolución del Parlamento Europeo en el documento Alfabetización de los medios de comunicación en un mundo digital en: www.europarl.europa.eu/sides/getDoc.do?pubRef=-//ep//text+ta+p6-ta-2008-0598+0+doc+xml+v0//es. 
- La educación mediática debe formar parte de la educación formal a la que tienen acceso todos los niños y ha de ser parte integrante de los planes de estudios de todos los niveles de educación escolar.

- La educación mediática debe tener, en la medida de lo posible, un carácter práctico y relacionar con materias económicas, políticas, literarias, sociales, artísticas e informáticas.

- El camino a seguir avanza hacia la creación de una materia específica, Educación Mediática, con un planteamiento pluridisciplinario vinculado a proyectos extraescolares.

- Recomienda a las instituciones de formación, como medida práctica, que fomenten la configuración de productos mediáticos (en el ámbito de los medios impresos, audiovisuales y nuevos) con la participación de alumnos y profesores.

$\mathrm{Y}$ es que la educación en medios de comunicación es una herramienta fundamental también para aprender a producir, no únicamente para aprender a ver, «implica claramente lectura y escritura», por lo que «debe comportar necesariamente tanto la interpretación como la producción de medios» (Buckingham, 2005: 90). Sugiere que sean los mismos alumnos quienes produzcan productos mediáticos propios con la ayuda de sus profesores, para desarrollar la dimensión práctica (Lull, 2008).

Sin lugar a dudas existe una tendencia en la sociedad de la información y el conocimiento hacia la educación de la ciudadanía en la recepción crítica de los medios de comunicación. Y, dentro de esa ciudadanía, los niños y jóvenes están siendo tomados en consideración. Para ellos se están creando, de modo ascendente, una serie de recursos didácticos con el fin de instruirlos en esta educación que era impensable en otros tiempos. Es lógico pensar que esta producción y análisis crítico de contenidos mediáticos forjen en los chicos una nueva forma de pensar, de actuar, de enfrentar aquellos medios que antes les eran ajenos y que ahora tienen en sus manos - por el momento- para aprender a descifrarlos. Por ello, no sería extravagante predecir el comportamiento de estos mismos niños el día de mañana. Cuando esos nativos digitales ${ }^{4}$ sean ya jóvenes y comiencen a producir verdaderos cambios en los medios, tal como desde ya están haciendo aún a nivel de principiantes.

Otro concepto que surge de todo este movimiento que se está generando en los campos de la comunicación y de la educación es el de las nuevas audiencias. Son aquellas que «participan en la producción mediática de manera innovadora en las estrategias y perspectivas de aproximación a los contenidos; en estilos novedosos en el tratamiento al arriesgar nuevas miradas a la realidad; con sentido universal en su relato y experimentales en la estructura narrativa y los modos de

4 Término acuñado por Marc Prensky que hace referencia a los niños que crecen utilizando en su cotidianidad las tecnologías de la comunicación. Consulte el artículo original en: www.marcprensky.com/writing/Prensky\%20\%20Digital\%20Natives, \%20Digital\%20Immigrants\%20-\%20Part1.pdf. 
contar» (Rincón, 2008: 97). Tal como también advierte Pérez-Tornero (1994), trabajar con los medios no es solo una actividad creativa, sino una necesidad, principalmente para la educación de la ciudadanía, puesto que interviene en aspectos sociales fundamentales como la convivencia y la democracia. «La alfabetización digital y mediática significa creatividad, participación y una nueva socialidad. Para muchos es una revolución que se está produciendo sin que nos demos cuenta... la juventud está haciendo una sublevación política sin que lo sepamos» $»^{5}$.

\section{Producción y visión crítica de los nativos digitales: una experiencia de campo}

La enseñanza específica de la producción audiovisual destinada al niño y adolescente abarca generalmente la escritura de guiones, conocer los procesos organizativos de una producción, el manejo de cámaras de vídeo, el funcionamiento básico del sonido y la iluminación, y la práctica de la edición digital. No obstante, en el ámbito de la lectura crítica no es infrecuente encontrar dificultades para medir sus capacidades de discernimiento ante los contenidos, sobre todo de la televisión. En primer lugar, debemos distinguir dos niveles de recepción crítica que podemos experimentar después del visionado de un programa de televisión. Pérez-Tornero (1994: 150-152) distingue entre la lectura crítica y la analítica. Para ejercitarnos en las operaciones y procesos de la lectura crítica hemos de ser capaces de: 1) Reconocer la finalidad pragmática del programa; 2) Discernir entre los niveles temáticos y narrativos; 3) Percibir el nivel formal; 4) Descubrir las relaciones co-textuales y contextuales; 5) Proponer alternativas; 6) Hacer una recreación distanciada del sentido del programa. Si se trata de una lectura analítica las operaciones entonces deberán ser profundizadas: 1) Realizar una lectura global del programa; 2) Fragmentarlo o dividirlo en partes diferenciadas; 3) Captar las distintas dimensiones del programa; 4) Reconocer su estructura; 5) Hacer una interpretación global. Seguir esta línea de investigación es intentar que el niño inicie su formación como telespectador consciente, crítico y activo.

Cabe recordar las definiciones del niño como sujeto en una investigación de este tipo. Llamamos «sujeto consciente» a aquél que reconoce el contenido y la técnica con que se realiza un programa de televisión, conoce el funcionamiento de la televisión por dentro, tiene una idea de lo que significa la industria de contenidos audiovisuales y distingue los tipos de audiencia que conforman la sociedad del espectador. El «sujeto crítico» es más bien aquél que sabe apreciar el contenido de un programa haciendo su propio juicio en cuanto a la finalidad del espacio en cuestión. Es decir, percibe las representaciones de la televisión como ideas producidas por personas, identifica el proceso de estructuración de

5 Parte de las conclusiones derivadas del Congreso Euro-Iberoamericano de Alfabetización Mediática y Culturas Digitales celebrado en mayo del 2010 en la Universidad de Sevilla (España). 
un espacio televisivo y se interesa por confrontar los valores que observa en él. Más que un analítico, que solo se forma con los años de práctica, la idea es motivar al niño hacia una lectura crítica del medio. Y, como «sujeto activo» se define al niño que utiliza su capacidad para proponer cambios sustanciales en los aspectos temáticos y/o técnicos de un programa de televisión. Podrá poner en práctica la lectura crítica del material producido por él o por su grupo de trabajo y será consciente de que puede ser activo ante el medio audiovisual (Pérez-Tornero, 1994: 147-162).

El estudio que presentamos fue desarrollado durante el curso 2005/2006 y trata sobre cómo los niños y niñas pueden aumentar su aprendizaje crítico sobre la televisión, si antes han estudiado y experimentado la práctica audiovisual. El objetivo general consistió en investigar el grado de conocimiento que puede alcanzar un pequeño grupo de niños entre 7 y 11 años al ser instruido con una guía didáctica, especialmente creada para esta experiencia de alfabetización audiovisual infantil. Los objetivos específicos fueron dos:

- Lograr que el grupo de niños experimente las operaciones de creación, producción y montaje de un documento audiovisual.

- Iniciar al grupo en la lectura crítica audiovisual de distintos géneros televisivos.

Para dar inicio a la investigación seguimos en primer lugar a Ander-Egg (1995: 130). Su procedimiento nos indicó que debíamos delimitar el Grupo Diana y una muestra, seleccionar las técnicas de recogida de datos más adecuadas, construir, en su caso, los instrumentos de observación y su prueba o pre test, e imaginar los posibles datos a obtener y los tipos de análisis que se pueden aplicar. Optamos, en esta ocasión, por el método de caso, seleccionando uno representativo del problema para estudiarlo y analizarlo posteriormente ${ }^{6}$. Por otra parte, este estudio - el documento audiovisual creado y analizado por niños- tiene cabida dentro de los estudios de naturaleza exploratoria ya que «responde a un tema desconocido, poco estudiado o novedoso» (Berganza, 2005: 54). Ahora bien, desde el enfoque del diseño de investigación se trataría también de un estudio «pre-experimental» de un solo grupo, con pre test y post test, esto es, «existe una situación base A que es comparada después de estar expuesta a un estímulo aplicado o situación B» (Sierra-Bravo, 1994: 144). Así, la muestra es medida previa y también posteriormente a la experiencia.

El universo de nuestra investigación -los niños en general-, se delimitó en población, con el factor delimitante de la edad: la llamada, por Piaget (1975: 61100), la «segunda infancia», 7 a 12 años, esto es, cuando inicia su desarrollo mental y entra de lleno en el sistema escolar, por lo que pasa a constituir la etapa de las «operaciones intelectuales». Nuestra muestra la constituyó un gru-

6 Puede consultarse el documento Las estrategias y técnicas didácticas en el rediseño: El estudio de casos como técnica didáctica que sintetiza el proceso de investigación para este tipo de diseño. Disponible en: www.itesm.mx/va/dide2/ documentos/casos.pdf [31-12-08]. 
po de 10 niños con edades comprendidas entre 7 y 11 años puesto que en la actualidad -a diferencia de los años 70 de cuando data el estudio de Piaget-los de 12 años ya están bastante inmersos en la pre-adolescencia. Se trató entonces de una experiencia participativa, con 52 horas lectivas de intervención didáctica, debates, diálogos y estudio del proceso evolutivo. El número de la muestra está justificado en el hecho de que cada una de las acciones de los niños ha de ser estudiada desde el punto de vista cualitativo. El proceso de la producción audiovisual abarca una serie de fases que incluyen numerosas acciones que sería difícil medir pormenorizadamente en un grupo más grande-, entre las que destacan: creación de la idea, definición y justificación del formato, escritura de guiones, diseño de storyboard, escritura del guión técnico, ubicación de localizaciones adecuadas, cálculo del presupuesto, reunión o «meeting» de producción, diseño del plan de producción, decisiones frente al tipo de iluminación y sonido más conveniente, actividades de regidor de plató, definición del tipo de vestuario, grabaciones, minutaje del material audiovisual, realización de la edición o montaje y la selección de efectos especiales. Este número de niños también permite la constitución de dos equipos de trabajo en los que usualmente participan en los talleres formado por: director-guionista, cámara, productor, técnico (encargado del sonido y la iluminación), editor. Son considerables las observaciones y otros tipos de control que se deben hacer tanto al grupo de niños como equipo de trabajo como a cada uno de los miembros que constituye la muestra. Por otra parte, ha de recordarse que se estudian dos campos de acción: la producción y otro no menos importante - como es la lectura crítica de distintos documentos audiovisuales, tal como se describe más adelante.

En cuanto al entorno familiar, era indispensable el diálogo con los padres, a fin de que tomaran parte activa de esta experimentación. El taller formó parte de una práctica de larga duración con visionados continuos, respuestas a cuestionarios, organización y producción de un documento audiovisual, entre otras tareas. En relación a los instrumentos de investigación, optamos en este caso, dada la naturaleza y el contexto del estudio, por la observación participante, método interactivo de recogida de información, que requiere una implicación del observador en los acontecimientos o fenómenos que está observando, y requiere de cierto aprendizaje que permita al investigador desempeñar el doble rol de observador y participante (Rodríguez-Gómez, 1996: 165). Buscamos con esta investigación los siguientes objetivos: 1) verificar que los niños de estas edades son capaces de producir un documento videográfico, siguiendo las fases de producción; 2) comprobar el grado de lectura crítica audiovisual adquirido. Para ello «se sondean las opiniones de los niños después del visionado de diferentes géneros mediante cuestionarios, minimizando los efectos del entrevistador, con las mismas preguntas y de la misma forma a cada persona» (Rodríguez-Gómez, 1996: 186). El cuestionario se presenta como el instrumento que nos ayuda a minimizar los efectos de la reiteración de interrogantes similares sobre un vídeo determinado. En el caso de los niños la repetición del esquema de preguntas hechas en forma oral no siempre funciona efectivamente. Por lo tanto, 
después de animarles a ver con atención cada uno de los géneros audiovisuales escogidos, procedimos a preguntar por cada aspecto del vídeo. El cuestionario estaba seccionado según el número de géneros audiovisuales seleccionados en la investigación: dibujos animados, series de televisión, películas y anuncios publicitarios.

Por otra parte, no descartamos otro instrumento de medición como la entrevista que, como herramienta de medición, aporta suficientes datos tanto en la fase inicial -pre test, para saber el nivel de conocimientos que tiene el grupo sobre la televisión, como en la fase final -post test-, para calibrar lo que más recuerdan del período de enseñanza teórico-práctico sobre la televisión. Por último, otra técnica que nos sirvió para descubrir reacciones de los niños en el grupo fue la cámara de vídeo. La grabación audiovisual constituyó una parte significativa en este trabajo porque se transformó en unos «nuevos ojos», que observaban la realidad que queríamos estudiar. Respecto al material didáctico, éste abarca una serie de guías basadas en el trabajo real de televisión e incluye un CD interactivo para el niño. Se trata de un recurso educativo diseñado en trabajos anteriores (Sánchez-Carrero, 2008; Aguaded, 1999), así que lo que queríamos era comprobar su utilidad en un grupo de niños de edades determinadas con atracción por el medio audiovisual. En cada guía se trata un tema diferente relacionado con una de las facetas de la producción audiovisual -preproducción, producción y postproducción- y se introduce a la lectura crítica de diversos géneros ${ }^{7}$ :

- Taller 1. El pequeño productor: Lectura crítica de dibujos animados.

- Taller 2. El pequeño cámara de TV: Lectura crítica de películas.

- Taller 3. El pequeño escritor de guiones: Lectura crítica de series de televisión.

- Taller 4. El pequeño mago del sonido y la luz: Lectura crítica de anuncios.

- Taller 5. El pequeño editor de vídeos: Lectura crítica de telediarios ${ }^{8}$.

En relación con las variables, procedimos en tres pasos básicos: asignarles un nombre, definirlas conceptualmente y, por último, operativamente, es decir, transformar la definición conceptual en actividades o tareas posibles de ejecutar. Las variables quedaron asignadas de esta manera:

- Variable 1: Producción audiovisual:

\footnotetext{
7 Los talleres que componen esta propuesta didáctica están recogidos en una publicación, que incluye un CD interactivo para el niño, titulada: Los secretos de la tele. Manual de alfabetización televisiva para niños y maestros (Sánchez-Carrero, 2009). Esta publicación recibió el apoyo financiero de la Consejería de Innovación y Ciencia de la Junta de Andalucía.

8 Este último apartado de recepción crítica no fue considerado para esta experiencia por razones de tiempo de aplica-
} ción del taller audiovisual infantil. 
- Definición conceptual. Producción audiovisual: es el proceso global que permite controlar las diferentes fases de realización de un documento audiovisual, a saber: preproducción, producción y postproducción.

- Definición operacional de la variable. Un grupo de niños estará capacitado para producir un documento audiovisual, si puede desarrollar las siguientes acciones del proceso: producción, guión, cámara, sonido e iluminación y edición de un vídeo.

- Variable 2: Lectura crítica audiovisual:

- Definición conceptual. Lectura crítica: es la capacidad de descubrir la finalidad y estructura de un documento audiovisual, llegando incluso a proponer cambios en dicho espacio.

- Definición operacional. Lectura crítica: se podrá afirmar que un niño ha realizado una lectura crítica elemental de un documento audiovisual, si reconoce aspectos temáticos, técnicos y propone cambios en su estructura, según corresponda al género visualizado.

En cuanto a elementos temáticos deberá reconocer: la historia, el argumento, los personajes, el rol protagónico, las características de los personajes, la intención del director, la solución que los personajes buscan a los problemas y la realidad de la fantasía. En relación con elementos técnicos deberá reconocer: los planos de cámara, los movimientos de cámara, las localizaciones, y además deberá ser capaz de proponer distintas combinaciones entre los elementos de un programa. Para medir la lectura crítica se requiere un recurso adicional, puesto que no se trata de conocimientos técnicos, que son más factibles de comprobación; sino de los distintos niveles de captación del sentido de una estructura narrativa. Para ello elaboramos un cuadro de diagnóstico exploratorio que nos sirvió para construir el contenido del cuestionario que debíamos aplicar de modo posterior al visionado de los vídeos elegidos por los propios niños. A continuación presentamos el cuadro correspondiente a cada género audiovisual.

a) Diagnóstico exploratorio para los dibujos animados

\begin{tabular}{|c|c|c|}
\hline Variable & Indicadores & ftems \\
\hline \multirow{8}{*}{$\begin{array}{l}\text { Lectura critica } \\
\text { de dibujos } \\
\text { animados }\end{array}$} & $\begin{array}{l}\text { Identifica los personajes del dibujo animado y su } \\
\text { caracteristica }\end{array}$ & $\begin{array}{l}\text { Rellena un cuadro con la identificación đe personajes y } \\
\text { su caracteristica principal }\end{array}$ \\
\hline & $\begin{array}{l}\text { Narra la historia de manera conereta sin contar } \\
\text { detalles insignificantes }\end{array}$ & Narra en poeas lineas el episodio de dibujos animados \\
\hline & Deseribe el comportamiento de los personajes & ¿Cuál es el comportamiento de los personajes? \\
\hline & $\begin{array}{l}\text { Identifiea la solución que los personajes dan a } \\
\text { sus problemas }\end{array}$ & $\begin{array}{l}\text { ¿Qué opinión tienes de la solueión que buscan los perso- } \\
\text { najes a los problemas? }\end{array}$ \\
\hline & $\begin{array}{l}\text { Justifica con argumentos el personaje que más le } \\
\text { gusta y el que menos }\end{array}$ & $\begin{array}{l}\text { ¿Cuál de los personajes te gustó más? ¿Por quê? } \\
\text { ¿Cuál de los personajes te gusto menos? ¿Por qué? }\end{array}$ \\
\hline & $\begin{array}{l}\text { Reconoce el propósito que querla transmitir el } \\
\text { autor del dibujo animado }\end{array}$ & ¿Cuảl crees que es el propósito del director? \\
\hline & $\begin{array}{l}\text { Propone cambios en el contenido el dibujo } \\
\text { animado }\end{array}$ & Si pudieras cambiar algo del video, $\iota q u e ́$ le cambiarias? \\
\hline & Diferencia la realidad de la fantasia & Lo que refleja el video es realidad o fantasia? ¿Por qué? \\
\hline
\end{tabular}


b) Diagnóstico exploratorio para las series de televisión

\begin{tabular}{|c|c|c|}
\hline Variable & Indicadores & ftems \\
\hline \multirow{10}{*}{$\begin{array}{l}\text { Lectura crítica } \\
\text { de serie de } \\
\text { televisión }\end{array}$} & $\begin{array}{l}\text { Narra la historia de manera concreta sin contar } \\
\text { detalles insignificantes }\end{array}$ & ¿De qué trata la historia? \\
\hline & Reconoce el tipo de mensaje que deja el capitulo & Qué tipo de mensaje te dejós? \\
\hline & $\begin{array}{l}\text { Identiflea algunos de los planos y movimientos } \\
\text { de cimara y su utilizacion }\end{array}$ & $\begin{array}{l}\text { Identifiea un plano movimiento de cámara y explica por } \\
\text { qué crees que lo utilizaron }\end{array}$ \\
\hline & $\begin{array}{l}\text { Recuerda fondos musicales y efectos sonoros y } \\
\text { su utilización }\end{array}$ & $\begin{array}{l}\text { ¿Había música? ¿De qué tipo? } \\
\text { ¿Recucrdas efectos sonoros? ¿Cuáles? ¿Por qué los } \\
\text { utilizaron? }\end{array}$ \\
\hline & $\begin{array}{l}\text { Reconoce escenografias y ambientacion en } \\
\text { interior o exterior }\end{array}$ & $\begin{array}{l}\text { ¿Qué ambientación predominaba: escenografia (interior) } \\
\text { o ambiente natural (exterior) }\end{array}$ \\
\hline & Describe el comportamiento de los personajes & ¿Cómo se comportaron los personajes? \\
\hline & $\begin{array}{l}\text { Detalla el vestuario de los personajes en moda y } \\
\text { colores }\end{array}$ & ¿Cómo visten los personajes? (moda y colores) \\
\hline & $\begin{array}{l}\text { Reconoce los titulos que aparecen en los eréditos } \\
\text { del capitulo }\end{array}$ & ¿Qué títulos aparecieron en los eréditos finales? \\
\hline & Diferencia la realidad de la funtasia & $\begin{array}{l}\text { ¿Crees que lo que viste en la serie puede suceder real- } \\
\text { mente? }\end{array}$ \\
\hline & $\begin{array}{l}\text { Reconstruye la historia cambiandole el final de } \\
\text { forma creativa }\end{array}$ & $\begin{array}{l}\text { Reconstruye la historia en pocas lineas pero cambiando } \\
\text { el final }\end{array}$ \\
\hline
\end{tabular}

\section{c) Diagnóstico exploratorio para las películas}

\begin{tabular}{|c|c|c|}
\hline Variable & Indicadores & frems \\
\hline \multirow{10}{*}{$\begin{array}{l}\text { Lectura critica } \\
\text { de pelieulas }\end{array}$} & Reconoce el tema esencial de la historia & ¿Cual es el tema principal de la historia? \\
\hline & $\begin{array}{l}\text { Distingue el rol protagónico de los personajes y } \\
\text { sus caracteristicas }\end{array}$ & $\begin{array}{l}\text { ¿Quién es y cómo es el protagonista principal? } \\
\text { ¿Quiénes son los protagonistas secundarios y cómo } \\
\text { ayudan al protagonista principal? }\end{array}$ \\
\hline & $\begin{array}{l}\text { Identifica el argumento que se utilizó para } \\
\text { desarrollar el tema }\end{array}$ & ¿Cual es el argumento de la pelieula? \\
\hline & $\begin{array}{l}\text { Reconoce el objetivo del protagonista y el } \\
\text { principal impedimento }\end{array}$ & ¿Qué desea el protagonista y qué se lo impide? \\
\hline & $\begin{array}{l}\text { Reconoce el lugar y la época en que transcurre la } \\
\text { historia }\end{array}$ & $\begin{array}{l}\text { ¿En qué época transcurre? } \\
\text { ¿Dónde ocurre la historia? }\end{array}$ \\
\hline & $\begin{array}{l}\text { Identifica la figura de héroe, villano, complice, o } \\
\text { autoridad y su comportamiento }\end{array}$ & $\begin{array}{l}\text { Rellena este cuadro y sefiala nombre y comportamiento } \\
\text { del héroe, villano, cómplice, autoridad, etc. }\end{array}$ \\
\hline & Diferencia la realidad de la fantasia & $\begin{array}{l}\text { ¿Crees que alguna parte de esta pelieula podria ocurrinte? } \\
\text { iPor que? }\end{array}$ \\
\hline & Identifica la intención del director & ¿Qué trató de expresar el director de la pelicula? \\
\hline & $\begin{array}{l}\text { Reconoce si la pelicula le ha dejado alguna } \\
\text { enseîanza y seilala los valores que destacan en } \\
\text { el film }\end{array}$ & $\begin{array}{l}\text { ¿Te ha enseñado algo la película? } \\
\text { ¿Cuáles son los valores que destacen en el film? }\end{array}$ \\
\hline & $\begin{array}{l}\text { Reconstruye la historia con los mismos persona. } \\
\text { jes y localizaciones de manera creativa }\end{array}$ & $\begin{array}{l}\text { Escribe en pocas líneas, con los mimos datos del video } \\
\text { (personajes y localizaciones) una historia diferente }\end{array}$ \\
\hline
\end{tabular}

\section{d) Diagnóstico exploratorio para los anuncios de televisión}

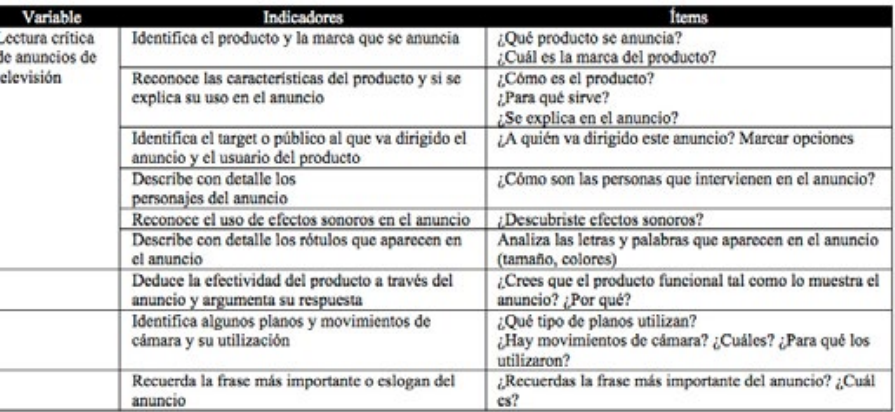

Una vez finalizado este proceso comenzamos a establecer la clasificación de los niveles de comprensión: «suficientemente crítico» (si cumplía con la totalidad de los indicadores antes señalados), «medianamente crítico» (si cumplía por lo 
menos con el 50\%), y «no crítico» (si no contestaba correctamente o si simplemente no respondía). Los niños de este estudio realizaron varias experiencias de producción audiovisual, demostrando su capacidad para escribir una historia (susceptible a convertirse en guión) y su posterior transformación en guión literario y técnico, así como organizar la producción, efectuar la grabación y editar en vídeo digital. Para ello, realizaron cuatro producciones propias de corta duración: «Magiakids», un espacio donde un mago hacía una exhibición de trucos; «Telediario Infantil», un reportaje deportivo destinado a un programa informativo; «Niños de la guerra», una animación en plastilina que narra la historia de un «niño soldado» que llega a matar a su propio hermano en medio de un ataque; y por último, «Telekids», un vídeo que resume el proceso de producción en un canal de televisión ficticio. En todos escribieron el guión, manejaron la cámara, planearon la producción (organización de grabaciones, búsqueda de localizaciones, elementos para escenografía, etc.) y, por último, acordaron la edición final de los vídeos.

\section{Gráfica 1: Actividades de la producción Magiakids}

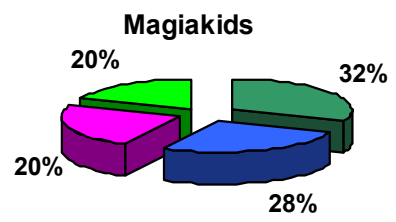

\begin{tabular}{|ll|}
\hline$\square$ Producción & $\square$ Cámara \\
$\square$ Guión & $\square$ Sonido e iluminación \\
\hline
\end{tabular}

Durante el proceso de preproducción de su primer programa «Megakids», su interés estaba enfocado en practicar los trucos de magia que presentarían, es decir, la producción del contenido. Más adelante, comenzaron a situar las cámaras y a ensayar los planos. El guión, sonido e iluminación no representaron una tarea difícil para el grupo en esta primera etapa. 


\section{Gráfica 2: Actividades de la producción Telediario Infantil de Deportes}

Telediario Infantil de Deportes

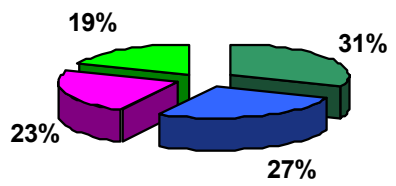

\begin{tabular}{|ll|}
\hline$\square$ Producción & $\square$ Cámara \\
$\square$ Guión & $\square$ Sonido e iluminación \\
\hline
\end{tabular}

En la segunda producción, estuvieron un poco más al tanto del guión pero descargaron todo el peso del programa en la presentadora. Igualmente establecieron el orden en lo que grabarían y el lugar, es decir, aspectos de la producción. Los asuntos técnicos estuvieron esta vez más descuidados. El cámara guardó dudas incluso hasta en la última toma de la grabación.

\section{Gráfica 3: Actividades de la producción Niños de la guerra}

Niños de la guerra

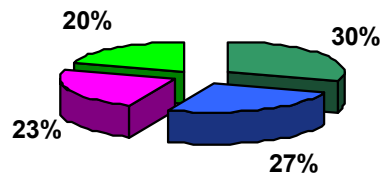

\begin{tabular}{|ll|}
\hline Producción & $\square$ Cámara \\
$\square$ Guión & $\square$ Sonido e iluminación \\
\hline
\end{tabular}

Esta producción, «Niños de la guerra», fue asumida por los chicos de mayor edad. Sin embargo, fue el equipo en pleno el que creó la idea y realizó la preproducción basada principalmente en el moldeado de las figuras en plastilina. Estuvieron cuidando las tomas y planos de cámara, pero no tanto la iluminación, que pudo mejorar la calidad del producto final. En cuanto al guión, puede afirmarse que fue la actividad más razonada aunque no se llevó a cabo tal como la planearon. 


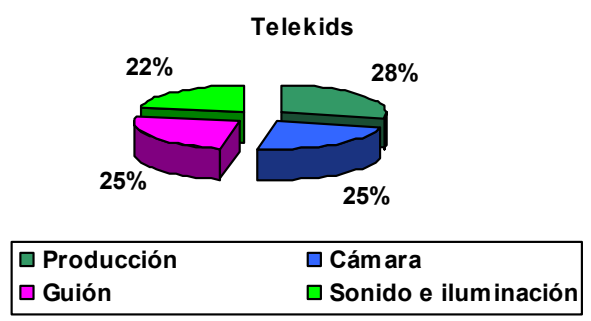

Esta última práctica de producción, «Telekids», nació como un ensayo en clave de humor pero luego se transformó en un espacio importante para todos. Prevaleció la idea de un guión explicativo con sus propias expresiones. El cámara recibió indicaciones del grupo mientras que el sonido constituyó el gran aprendizaje puesto que realizaron prácticas en las que el micrófono no captaba el audio por el mal uso que hacían de él.

Sin duda, el grupo evidenció importantes progresos. Las actividades específicas de producción fueron siempre las que alcanzaron el máximo nivel de entendimiento. El aspecto de más fácil comprensión fue la fase de producción. Todos aceptaron que la experiencia requería avanzar paso a paso y una mayor organización para poder efectuar la grabación: búsqueda de permisos, localizaciones, ensayos, maquillaje, etc. Esto se comprueba sobre todo en la última experiencia. En cuanto al manejo de la cámara hay que destacar que mejora considerablemente después de la segunda producción. Esto se observa conretamente cuando salen del pequeño plató improvisado dentro de la sala de clase para visitar exteriores, aumentando su interés por los detalles de cámara: planos, movimientos... No sucedió lo mismo con la escritura de guiones pues desde la primera grabación se ocuparon de los textos de los presentadores. Sonido e iluminación constituyeron el último tema de su interés. No es sino cuando comienzan a visionar el material grabado que se percatan de errores en el sonido por ausencia de micrófono o por mala ubicación. Hay que señalar que éstos son aprendizajes técnicos especializados, que no son determinantes en esta primera etapa de enseñanza audiovisual.

Finalmente, la producción de un cortometraje de ficción conformó nuestro post test. Titulado por ellos, «El secreto de Juanito», cuenta la historia de un niño maltratado por los padres de la niña protagonista (Lola), quien finalmente logra concienciarlos de su error. En esta ocasión los niños de menor edad (7 años) presentaron menos efectividad a la hora de realizar tareas como el guión o incluso algunos usos de cámara, pero actuaron con más entusiasmo en el diseño del Storyboard y en la fase de edición. Los niños de 8 y 9 años estuvieron más atentos a las áreas de guión y de sonido e iluminación. Justamente, una 
de las chicas de 8 años fue la creadora de la historia original del cortometraje, incluso debatió con sus compañeros su propuestas y el perfil de los personajes. En cuanto a los mayores del grupo (10 y 11) asumieron todas las actividades de producción en general con responsabilidad, se hicieron cargo del guión, la cámara y la edición final.

En relación con la segunda variable, la lectura crítica, observamos diversos resultados que muestran el desarrollo del grupo en el visionado de dibujos animados, series de televisión, películas y anuncios publicitarios audiovisuales.

\section{Comparativa en visionado de dibujos animados}

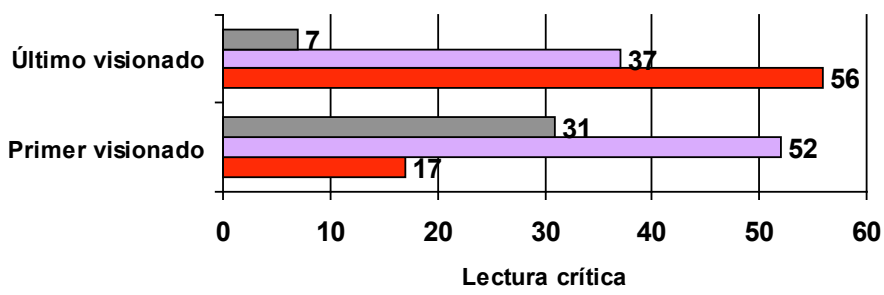

$\square$ Suficientemente crítico $\square$ Medianamente crítico $\square$ No crítico

\section{Comparativa en visionado de series de TV}

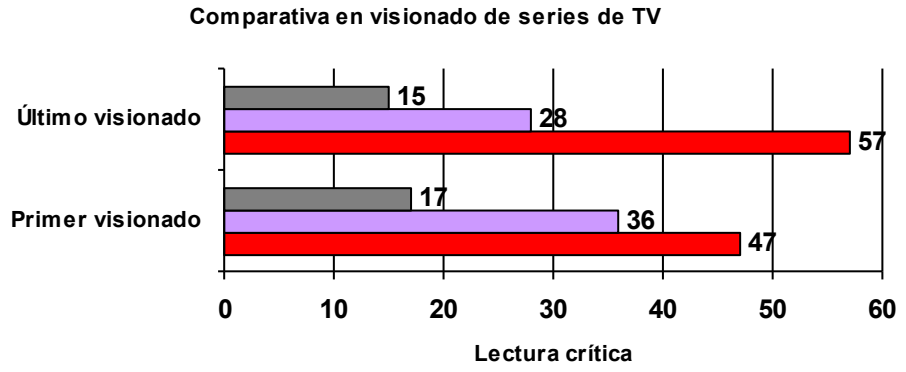

$\square$ Suficientemente crítico $\square$ Medianamente crítico $\square$ No crítico 


\section{Com parativa en visionado de películas}

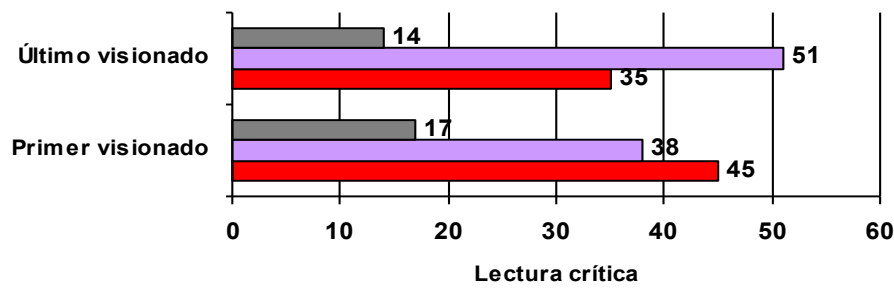

$\square$ Suficientemente crítico $\square$ Medianamente crítico $\square$ No crítico

Com parativa en visionado de anuncios de TV

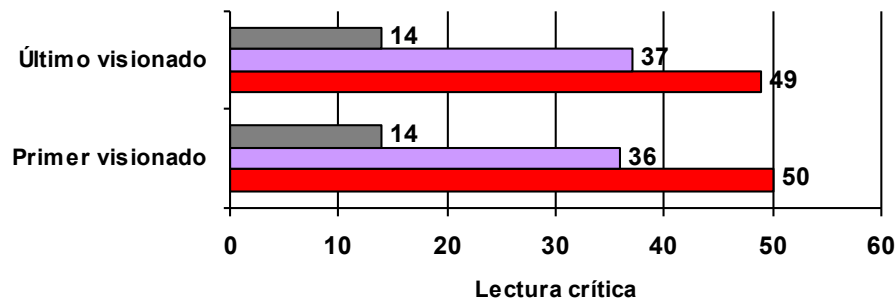

Suficientemente crítico $\square$ Medianamente crítico $\square$ No crítico

\section{Visionado corre lativo en el aprendizaje de}

la lectura crítica audiovisual

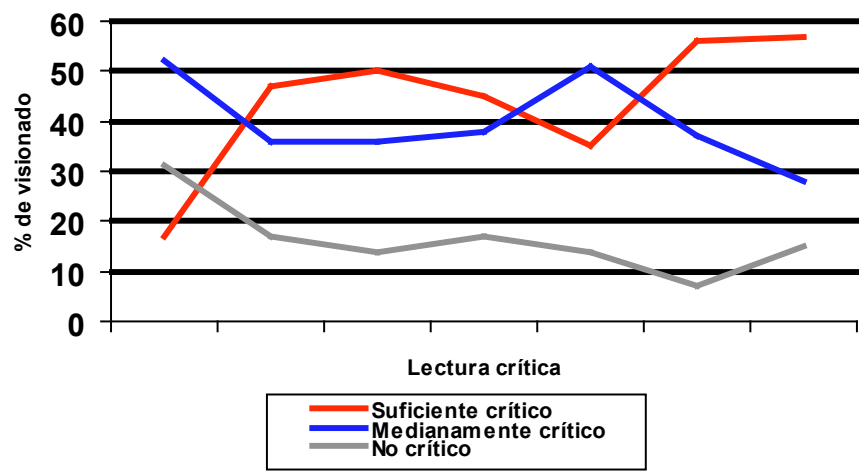


Observando la evolución del grupo en lectura crítica, podemos afirmar que durante el proceso de formación los niños aumentaron su nivel de criticidad ante la televisión. La categoría «no crítica» disminuyó en casi la mitad (48\%) desde el comienzo al final, mientras que el grado «suficientemente crítico» se triplicó durante todo el proceso de aprendizaje. Este comportamiento (disminución de los «no críticos» y aumento de los «suficientemente críticos») revela un progreso, y por lo tanto puede considerarse como un resultado positivo en el proceso de lectura crítica audiovisual infantil. Por otra parte, el nivel «medianamente crítico» se redujo aproximadamente a la mitad (53\%) durante el desarrollo de la experiencia. Este hecho resulta significativo puesto que el grupo fue superando las escasas intervenciones de las primeras sesiones para comenzar a debatir y hacer juicios argumentados en relación con los espacios televisivos.

En el visionado de películas la muestra presentó un mayor rango de porcentaje en el grado «medianamente crítico», de $38 \%$ a $51 \%$. Si bien es cierto que desciende el nivel de criticidad suficiente o alto, también baja el grado «no crítico», de $17 \%$ a $14 \%$. Las complicaciones las encontraron en este caso a la hora de reconocer si las situaciones presentadas podían suceder en la vida real. También se prestaron a confusión las controversias acerca de los distintos valores (verdad, honestidad, etc.), presentes en los argumentos fílmicos. Curiosamente no detectamos muchas diferencias entre el primero y el último visionado de lectura crítica en anuncios publicitarios. La mitad del grupo de niños resultó «suficientemente crítico» desde el primer visionado y así se mantuvo prácticamente igual hasta el final. Solo el $14 \%$ de las respuestas fueron «no críticas». Observamos su facilidad para desaprobar las incitaciones a la compra y no dejarse persuadir tan fácilmente por la mayoría de los spots. Tenían interiorizado el hecho, presumiblemente aprendido en sus hogares, que reza «la publicidad es mentira», fundamentado principalmente en las conversaciones familiares. En conclusión, con este estudio demostramos que los chicos tienen capacidad para desestructurar los documentos audiovisuales una vez realizadas las prácticas de producción audiovisual guiada.

En la primera fase los niños no tenían clara la estructura básica del cuento, solo dos de ellos diferenciaba entre fantasía y realidad en televisión, y ninguno conocía nada sobre aspectos técnicos ni de producción audiovisual. Al final de la aplicación didáctica el grupo logró crear una idea que contaba con los requisitos para ser transformado en un documento audiovisual, es decir, con posibilidades de ser adaptada en un guión literario y posteriormente en uno técnico. De la misma forma fue capaz de cumplir las actividades de preproducción y producción, preparando las tareas, grabando las distintas escenas y utilizando adecuadamente el equipamiento técnico que tenía a su alcance. Por último, a pequeña escala, se adquirió la capacidad para realizar el montaje audiovisual del material grabado, convirtiendo su historia en un cortometraje de cinco minutos destinado a un público infantil y familiar. Por otra parte los niños y niñas adquirieron claves para una lectura crítica básica, pudiendo seccionar episodios 
de dibujos animados, capítulos de series, extractos de películas o anuncios de televisión. De igual manera, los niños fueron competentes en el momento de hacer crítica a su trabajo final, llegando a reconocer errores tanto en el argumento como en el aspecto técnico y proponiendo soluciones factibles para mejorar ese producto audiovisual. Respecto a la producción audiovisual con cámaras se detecta que los niños necesitan tiempo para explorar los equipos básicos (cámara, trípode, ordenador, programa de edición...). Si bien en un principio parecía suficiente la práctica de planos y movimientos cuando se les asignaba durante las clases, fue necesario antes del proceso de producción, dejarles sesiones completas para que experimentaran con la cámara. Así, notamos su curiosidad por conocer la funcionalidad de las distintas prestaciones de la cámara y la inquietud por conocer si podían grabar ellos mismos.

En cuanto a la lectura crítica observamos que el grupo pudo aguzar sus sentidos visual y auditivo: mostraron interés por conocer cómo se lograban los efectos especiales, tanto de sonido como los visuales y de series como de películas. También demostraron su compañerismo al querer trabajar con los demás. Si los dejábamos actuar individualmente no tardaban mucho en conectar con alguien más y mostrar o bien el resultado de su trabajo o buscar la aprobación de alguna decisión tomada. En las entrevistas se percibe el grado de entusiasmo por el mundo audiovisual. Al llegar, los niños tenían curiosidad, pero a la vez se mostraban tímidos ante la cámara y en algunos casos, rechazo al verse grabados e incluso observados por sus compañeros. Contrariamente, al final, fueron ellos mismos quienes animaban a sus compañeros a grabar alguna idea por rápida y esencial que fuera. Se volvieron observadores de la televisión con enfoque crítico, hecho que corroborado por las madres que acudieron a la sesión final. Una de ellas argumentó que su hijo estaba mucho más sociable y que le enseñaba ciertos aspectos técnicos como la situación de las cámaras en el plató o el tipo de plano o movimiento en series o anuncios de televisión. Otra señaló que el niño le animaba a que dejara de ver «los programas de cotilleo» y que incluso apagaba con más frecuencia el aparato de televisión. El resultado de esta experimentación no es más que otro eslabón en la cadena de prácticas de educación mediática que se vienen desarrollando en las últimas décadas en diversas latitudes del mundo.

El uso de medios digitales para desarrollar habilidades no referidas al campo de la comunicación por parte de los más pequeños también puede verse reflejada en otras investigaciones. Un ejemplo de ello es el estudio titulado YouTube y el desarrollo de las competencias básicas. Una experiencia de investigación en el aula, dirigida por Antonia Ramírez García, de la Universidad de Córdoba (España). En este caso se trata el estudio se desarrolló durante el curso 2008/09 en el CEIP Gran Capitán en Montilla (Córdoba), y en otros cuatro centros educativos. La investigación, calificada como cuasi-experimental, consta de tres unidades didácticas aplicadas a un grupo de alumnos de cuarto de educación primaria. Los resultados fueron medidos, al igual que en el caso descrito anteriormente, a 
través del pre test y el post test de un grupo control y de otro experimental. En una de las unidades didácticas se utilizó un vídeo de YouTube como elemento en torno al cual se plantearían las tareas de la actividad relacionadas con la asignatura específica de Matemáticas. La utilización de la Web 2.0 mostró que el uso del vídeo digital contribuyó a aumentar el nivel de competencia del alumnado del grupo experimental y ayudó a atender a la diversidad de capacidades e intereses de la muestra. Además de obtener éstos y otros objetivos, la investigación sirvió para desarrollar una metodología de acción en el aula a través del diseño de cuadernos de trabajo para el alumno adaptados a los distintos niveles curriculares existentes: básico, medio y avanzado. Todo esto quedó patente en los resultados post-experimentales, tal como señala su autora: «El alumnado al que se le aplica el programa formativo (grupo experimental) alcanza unos mejores rendimientos académicos en el área de Matemáticas que el alumnado perteneciente al grupo control» (Ramírez, 2010: 11).

\section{El empoderamiento de la «Generación M2»}

«Generación M2» es un término que nace en los Estados Unidos para definir a los chicos entre 8 y 18 años que utilizan los medios digitales como entretenimiento. Según el informe de la Fundación Kaiser Family, el tiempo que estos niños y adolescentes pasan frente a esos medios se ha incrementado en una hora y 17 minutos en los últimos cinco años. El documento titulado Generación M2: los medios en la vida de los jóvenes de 8 a 18 años ${ }^{9}$, realizado entre octubre de 2008 y mayo de 2009 con la participación de más de 2000 chicos, atribuye este aumento al fácil acceso que tienen a dispositivos móviles -que son a la vez plataformas multimedia- tales como los teléfonos celulares y los iPod. El informe también implica a las redes sociales como Facebook y Twitter entre la serie de causas de este acrecentamiento en el consumo de los medios como ocio por parte de los más jóvenes. Entre sus actividades de ocio más usuales destacan: el visionado de televisión -en cualquiera de las plataformas-, el uso de videojuegos, escuchar música en reproductores mp3, realizar actividades de distinta índole en Internet y comunicarse a través del flujo de mensajes electrónicos utilizando la telefonía móvil. No obstante, una parte de esos chicos que a partir de los 8 años de edad comienzan a utilizar los medios como entretenimiento está produciéndolos, aún sin conocer a ciencia cierta sus funcionalidades ni tampoco los riesgos de una sobreutilización.

En este sentido, es interesante reseñar el hecho de que el portal YouTube ha incorporado en junio de 2010 un editor de vídeos online con el propósito de que el usuario pueda editar allí sus propios vídeos ${ }^{10}$. Esta herramienta facilita al usuario la producción audiovisual y su emisión por la Red puesto que puede

$\overline{9}$ El informe puede consultarse en: www.kff.org/entmedia/upload/Executive-Summary-Generation-M-Media-in-theLives-of-8-18-Year-olds.pdf.

10 YouTube presenta su editor online en: www.youtube.com/user/YouTube?blend=2\&ob=1. 
combinar, recortar, añadir bandas sonoras y, en general, crear nuevos vídeos sin ocuparse del problema de los formatos. La convergencia de medios también ha favorecido esa nueva faceta de niños y jóvenes no solo desde el papel de usuario sino también en el rol de aprendices y desarrolladores de medios digitales tales como blogs, cortometrajes o periódicos en la web. Y es esto, justamente, lo que les está dotando de un empoderamiento no vivido por generaciones anteriores. Por ello, hoy más que nunca, se requiere del establecimiento definitivo de la educación mediática en la escuela, de tal forma que favorezca su complementación en otros entornos como el familiar y el propio medio de comunicación.

Tal como señala Tyner, los medios de comunicación son un aspecto importante en la vida del ciudadano. Las personas con más problemas de aprendizaje son aquellas que ven más televisión y películas en DVD, así que potenciar su aprendizaje crítico es esencial. Los textos, los contenidos y los contextos son decisivos: el entorno familiar, el mediático y el educativo ${ }^{11}$. Por otra parte, las prácticas de alfabetización mediática están siendo influenciadas por la proliferación de los medios emergentes. Tyner apunta a la escuela como responsable de la creación de sistemas innovadores que estén diseñados para adaptar las prácticas de alfabetización a los estudiantes. «Los estudios de casos muestran claros ejemplos donde se integran el uso de redes sociales interculturales, la visualización de datos, los videojuegos, las simulaciones y los mundos virtuales en el aprendizaje interdisciplinario en los espacios de educación informal, como por ejemplo las actividades extraescolares...» (Tyner, 2010: 1). En contraposición, la educación formal continúa rezagada en cuanto al uso de herramientas digitales y su nueva alfabetización. Debemos recordar que la alfabetización mediática ha de abarcar los nuevos medios y los anteriores también, los que siguen siendo consumidos por la gran mayoría de menores en el mundo como son el cine y la televisión, visionados en cualquiera de las plataformas. No empeñarnos en perseguir con afán únicamente una educación por los nuevos medios -como blogs y telefonía móvil-, cuando todavía no hemos implementado acciones para los otros. Indudablemente se trata de un campo que crece desmesuradamente por lo que hemos de actuar con la mayor precisión y prontitud posibles.

Lo cierto es que urgen acciones concretas que ayuden a avanzar en el campo de la educación mediática. Un modelo a seguir es la Cumbre Mundial de los medios por los niños y jóvenes ${ }^{12}$, celebrada en Karlstad (Suecia) en junio de 2010, en el cual se busca responder a inquietudes fundamentales como ¿qué están haciendo los niños con los medios? y ¿qué están haciendo los medios con los niños? Este foro -que se efectúa cada tres años desde 1995- supone no solo el encuentro de importantes expertos en el área de la educación y la comunicación sino una oportunidad para que niños y jóvenes muestren sus ideas en relación con el uso que hacen de los medios digitales. Hasta Escandinavia se han trasladado

11 Parte de la intervención de Kathleen Tyner en el Congreso Euroiberoamericano de Alfabetización Mediática y Culturas Digitales.

12 www.wskarlstad2010.se. 
chicos de gran parte del mundo para participar, algunos de forma activa como el equipo de adolescentes que produce un diario para el sitio web de la Cumbre. Ellos entrevistan, fotografían y redactan pequeñas notas informativos sobre lo que acontece en el evento. Otro ejemplo es el de Yorka, una niña boliviana de 13 años -proveniente de una familia de mineros- que viajó hasta Karlstad para presentar su documental sobre la basura, que ya había ganado un premio en el Festival Kolibrí de su país. La pequeña cineasta se agranda al hablar de su película cuyo tema afecta seriamente a su región. Será presentada frente a otros muchos chicos que también están viviendo el proceso de empoderamiento que les brinda el hecho de producir en un universo digital.

Eventos como éste han de multiplicarse. Con ellos se busca lograr una mayor comprensión de la evolución de los medios de comunicación para niños y buscar fórmulas para mejorar calidad de los contenidos. Es fundamental no solo llamar la atención de los agentes clave en la difusión de este tipo de foros sino ayudar también a los países en vías de desarrollo a ofrecer a los niños y jóvenes oportunidades en sus medios de comunicación. Para obtener información de otras múltiples experiencias e iniciativas, se puede consultar la web de la ONU (Alianza de las Civilizaciones) (www.aocmedialiteracy.org), donde se explica el compromiso de los organismos internacionales, como también hemos visto al inicio de este estudio con el Parlamento Europeo, en recomendar que la educación mediática sea parte esencial en los currículos escolares y no formales, como parte de un compromiso mundial para la formación de unos espectadores activos y críticos en todo el mundo.

\section{Referencias}

Aguaded, José Ignacio (1999). Descubriendo la caja mágica. Nos y aprendemos a ver televisión. Huelva: Grupo Comunicar.

Aguaded, José Ignacio y Contin, Silvia (Dirs.) (2002). Jóvenes, aulas y medios de comunicación. Buenos Aires: La Crujía.

Ander-Egg, Ezequiel (1995). Técnicas de investigación social. Buenos Aires: Lumen.

Berganza, María Rosa (2005). Investigar en comunicación. Madrid: McGraw Hill.

Buckingham, David (2005). Educación en medios. Alfabetización, aprendizaje y cultura contemporánea. Barcelona: Paidós.

Lull, James (2008). Los placeres activos de expresar y comunicar. En: Comunicar, $\mathrm{n}^{\circ} 30 ; 21-26$.

Pérez-Tornero, José Manuel (1994). El desafío educativo de la televisión. Barcelona: Paidós Comunicación. 
Piaget, Jean (1975). Seis estudios de psicología. Barcelona: Seix Barral.

Ramírez, Antonia (2010). You Tube y el desarrollo de competencias básicas. Una experiencia de investigación en el aula. En: Congreso de Alfabetización Mediática y Culturas Digitales. Sevilla: Asociación de Televisiones Educativas y Culturales de Iberoamérica y Universidad de Sevilla, 1-12.

Ricón, Omar (2008). No más audiencias, todos devenimos productores. En:Comunicar, nº $30,93-98$.

Rodríguez-Gómez, Gregorio (1996). Metodología de la investigación cualitativa. Málaga: Aljibe.

Sánchez-Carrero, Jacqueline (2009). Los secretos de la tele. Manual de alfabetización televisiva para niños y maestros. Sevilla: Aconcagua.

Sánchez-Carrero, Jacqueline (2008). Pequeños directores. Niños y adolescentes creadores de cine, vídeo y televisión. Sevilla: Aconcagua.

Sierra-Bravo, Restituto (1994). Técnicas de investigación social. Madrid: Paraninfo.

Tyner, Kathleen (2010). Media Literacy. New Agendas in Communication. Texas: Routledge.

\section{Netgrafía}

Alfabetización de los medios de comunicación en un mundo digital. Disponible en: www.europarl.europa.eu/sides/getDoc.do?pubRef=-//ep//text+ta+p6-ta2008-0598+0+doc+xml+v0//es (18/06/10).

Center for Media Literacy. Disponible en: www.medialit.org. [Última consulta el 18/06/10]

Digital Natives, Digital Inmigrants. Disponible en: www.marcprensky.com/writing/Prensky\%20-\%20Digital\%20Natives,\%20Digital\%20Immigrants\%20-\%20 Part1.pdf (18/06/10).

El estudio de casos como técnica didáctica. Disponible en: www.ulavirtual.cl/ ulavirtual/SITIO\%20WEB\%20CRA/recursos_ensenanza/estrategiasytecnicasdidacticas/estudio_de_casos_como_tecnica_didactica.pdf (18/06/10).

El PE sugiere introducir una asignatura de Educación mediática en las escuelas europeas. Disponible en: www.europarl.europa.eu/news/expert/infopress_ page/037-44615-350-12-51-906-20081216IPR44614-15-12-2008-2008-false/default_es.htm (18/06/10):

Generation M2. Media in the lives of 8-18 Years-old. Disponible en: www.kff. org/entmedia/upload/Executive-Summary-Generation-M-Media-in-the-Lives-of8-18-Year-olds.pdf (18/06/10). 
World Summit on Media for Children and Youth. Disponible en: www.wskarlstad2010.se (18/06/10).

You Tube Broadcasting Ourselves. Disponbile en: www.youtube.com/user/ YouTube?blend=2\&ob=1 $(18 / 06 / 10)$.

\section{Referencia de este artículo}

Aguaded Gómez, José Ignacio y Sánchez Carrero, Jacqueline (2013). El empoderamiento digital de niños y jóvenes a través de la producción audiovisual. En: adComunica. Revista Científica de Estrategias, Tendencias e Innovación en Comunicación, $\mathrm{n}^{0} 5$. Castellón: Asociación para el Desarrollo de la Comunicación adComunica, Universidad Complutense de Madrid y Universitat Jaume I, 175-196. DOI: http://dx.doi.org/10.6035/2174-0992.2013.5.11 